\title{
Presence of secondary bladder cancer following radical nephroureterectomy for upper tract urothelial carcinoma: Characteristics, Risk Factors, and Predictive Value
}

\section{Shicong Lai ( $\nabla$ sclai2015@pku.edu.cn )}

Graduate School of Peking Union Medical College and Chinese Academy of Medical Sciences https://orcid.org/0000-0001-5770-2861

\section{Pengjie Wu}

Beijing Hospital

Shengjie Liu

Beijing Hospital

\section{Samuel Seery}

Chinese Academy of Medical Sciences and Peking Union Medical College

Jianyong Liu

Beijing hospital

Lei He

Beijing Hospital

Ming Liu

Beijing hospital

Yaoguang Zhang

Beijing hospital

Jianye Wang

Beijing Hospital

\section{Research article}

Keywords: Cancer-specific survival, Intravesical recurrence, Radical nephroureterectomy, Secondary bladder cancer characteristics, Upper urinary tract urothelial carcinoma

Posted Date: January 14th, 2021

DOI: https://doi.org/10.21203/rs.3.rs-143706/v1

License: (c) (i) This work is licensed under a Creative Commons Attribution 4.0 International License. Read Full License 
Page $2 / 17$ 


\section{Abstract \\ Objective}

To assess the characteristics, predictive risk factors, and prognostic effect of secondary bladder cancer (BCa) following radical nephroureterectomy (RNU) in upper tract urothelial carcinoma (UTUC).

\section{Methods}

Using the Surveillance, Epidemiology, and End Results (SEER) database, the authors analyzed clinicopathologic characteristics and survival data from 472 UTUC patients with secondary BCa after RNU between 2004 and 2017. Cox's proportional hazard regression model was implemented to identify independent predictors associated with post-recurrence outcomes. The threshold for statistical significance was $p<0.05$.

\section{Results}

In total, 200 Ta-3NOMO localized UTUC patients with complete data were finally included. With a median follow-up of 71 months (interquartile ranges [IQR] $36-103.75$ months), 52.5\% ( $n=105)$ had died, with $30.5 \%(n=61)$ dying of UTUC. The median time interval from UTUC to BCa was 13.5 months (IQR 640.75 months). According to multivariable Cox regression analysis, patients with intravesical recurrence (IVR) located at multiple sites, advanced BCa stage, higher BCa grade, elderly age and a shorter recurrence time, encountered worse cancer-specific survival (CSS) (all $p \llbracket 0.05$ ).

\section{Conclusions}

For primary UTUC patients experiencing IVR after radical surgery, advanced age, multiple IVR sites, shorter recurrence time, higher $\mathrm{BCa}$ stage, and grade proved to be significant independent prognostic factors of CSS. We ought to pay more attention to IVR prevention as well as to earlier signs which may increase the likelihood of early detection. Having the ability to manage what may be seen as the superficial BCa signs may enable us to improve survival but further research is required.

\section{Introduction}

Upper urinary tract urothelial carcinoma (UTUC) is a lethal malignancy which has characteristic synchronous or metachronous multifocal recurrences throughout the genitourinary tract [1-3]. Radical nephroureterectomy (RNU) with bladder cuff excision (BCE) remains the gold standard surgical intervention for non-metastatic UTUC; however, there are still 20-50\% who develop intravesical recurrence (IVR) following radical surgery throughout their lifetime [4]. Some have even reported that more than $60 \%$ of those undergoing RNU will develop IVR postoperatively within two years. 
Numerous studies have been designed to identify the relevant IVR predictors after RNU. However, little detailed data around the clinicopathologic characteristics, predictive risk factors, and prognostic effect of secondary bladder cancer (BCa) in UTUC exists. Yamashita et al. and Elalouf et al. found that patients with IVR after RNU had worse cancer-specific survival (CSS) and indeed overall survival $[5,6]$. Unfortunately, this evidence comes from seemingly disparate studies, involving relatively few participants which inhibits our ability to provide strong recommendations. Hou et al. once developed a prognostic nomogram using a clinical data set garnered from the surveillance, epidemiology and end results (SEER) database between 2010 and 2015 [7]. However, despite the analytical sophistication involved in their study, it suffered substantial recruitment bias and incomplete individual patient data which decreases the value and reduces reliability. Additionally, a large proportion of UTUC patients included in their final sample did not undergo bladder cuff excision (BCE) which may actually predispose patients to local recurrences $[8,9]$.

Complete information around histopathological characteristics is necessary if we are to develop a reliable evidence-base to guide best clinical practice. However as yet, few have managed to design, conduct, report and disseminate investigations which garner real insight for clinical practice. As such, it remains necessary to conduct a rigorous assessment of factors associated with post-recurrence outcomes in primary UTUC patients experiencing IVR following radical surgery.

\section{Materials And Methods}

\section{Data source and study population}

After approval from the National Cancer Institute (NCI), patients diagnosed with UTUC (renal pelvis or ureteral) between 2004 and 2017 were identified from nine SEER cancer registries. The site codes C65.9 and C66.9 as well ass histological codes taken from the $3^{\text {rd }}$ edition of International Classification of Diseases for Oncology (i.e., 8120, 8122, 8130, and 8131) were used to identify patients. All selected patients underwent RNU with BCE and subsequently had received secondary BCa diagnosis.

Patients were deemed ineligible if they met any of the following criteria: (1) Patients with synchronous or previous BCa when diagnosed with UTUC; (2) Patients with bilateral UTUC; (3) Patients with distant metastatic UTUC or only with lymph node metastasis; (4) patients who had not undergone radical surgery or had received RNU without BCE; (5) Patients whom did not develop secondary BCa post-surgery; and (6) Patients with insufficient clinicopathological information or incomplete survival data (e.g., ethnicity, gender, tumor location, tumor stage, grade, architecture, laterality, cause of death, interval between UTUC and $\mathrm{BCa}$, length of survival, and unknown length of follow-up).

Since the SEER database is publicly accessible and patient information was anonymous, this study does not require informed consent. However, current study conformed to the 1964 Helsinki Declaration (and the more recent amendments) and was performed in accordance with the ethical standards of the institutional and national research committee. 


\section{Definition of variables and follow-up}

All prerequisite information was abstracted from SEER database. Basic demographics including age, gender, race, the time interval from UTUC to IVR, and tumor characteristics (i.e., tumor location, stage, grade and architecture) of both UTUC and BCa were carefully retrieved. As tumors histopathological grades were classified as four types (i.e., well diferentiated, moderately diferentiated, poorly diferentiated, and undiferentiated) in the SEER database, we defined the well and moderately differentiated as low grade, and defined the poorly and undifferentiated type as high grade.

The locations of secondary $\mathrm{BCa}$ were also divided into three sites: surgical site, which refers to $\mathrm{BCa}$ located in BCE field (i.e, the bladder trigone or posterior wall) and/or open excision filed (anterior wall) (Figure 1A); non-surgical site, which refers to BCa located in the bladder dome, neck or later wall (Figure 1B); whilst multiple sites was defined as the synchronous presence of two or more pathologically confirmed BCa (Figure 1C).

Recurrence time was defined as the time interval between UTUC and IVR. CSS was defined as the time from diagnosis until death due to UTUC, which was defined according to SEER-specific cause of death coding (i.e., code 29020). Data from patients who died of other causes and those who survived to the final follow-up point were classified as censored for analytical purposes.

\section{Statistical analysis}

Categorical variables are presented as frequencies and proportions, while continuous variables are expressed as medians with interquartile ranges (IQR). Survival curves were generated using the KaplanMeier method and multivariate survival analysis was assessed using Cox's proportional hazard regression model, which is a forward step likelihood ratio based model used to identify independent risk factors associated with mortality. All analyses were conducted using the Statistical Package of Social Sciences for Windows, version 20 (SPSS, Chicago, IL). P values lower than 0.05 were considered statistically significant.

\section{Results}

\section{Baseline characteristics of the study population}

A total of 200 Ta-3NOMO localized UTUC patients met the predetermined eligibility criteria were eventually included in the final analysis. Detailed patient demographics and clinicopathological characteristics are presented in Table I. In this instance, the majority of this sample were caucasian (91.0\%). Overall, 132 $(66.0 \%)$ patients had renal pelvic tumors and $68(34.0 \%)$ with ureteral tumors. High tumor grade was identified in $75.5 \%(n=151)$ patients. The pathologic stage distribution of UTUC in this cohort was $45 \%$ $(\mathrm{n}=90)$ pTa-1, with $21.5 \%(\mathrm{n}=43)$ pT2, and 33.5\% $(\mathrm{n}=67)$ pT3.

Of the 200 patients in the entire cohort, the median recurrence time was 13.5 months (IQR 6-40.75 months). Secondary BCa were located as follows, with $21 \%(n=42)$ and $26.5 \%(n=53)$ positioned at site I 
and II respectively. The remaining 52.5\% ( $\mathrm{n}=105)$ who had multifocal tumors residing in site III. High tumor grade was found in 59\% $(n=118)$ patients. Pathologic stage distribution highlighted that Noninvasive bladder cancer (NIVBC) (pTa-1) and IVBC (pT2-4) was found to be $90 \%(n=180)$ and 10\% $(n=20)$, respectively.

\section{Survival outcomes and predictors}

At the median follow-up of 71 months (IQR $36-103.75$ months), 52.5\% ( $n=105)$ had died, of whom 30.5\% $(n=61)$ had died of UTUC. As can be seen in Table 2 and Figure 2, both Kaplan-Meier and univariable Cox regression analyses suggested that patient age, UTUC stage, BCa stage, BCa grade, BCa location, and recurrence time are significant, independent predictors of CSS. However, under multivariable Cox regression analysis, UTUC tumor stage was not an independent predictive factor of CSS.

An advanced BCa stage (hazard ratio [HR]:2.900; 95\% confidence interval [Cl]:1.441-5.839; $p=0.003$ ) and higher BCa grade ((HR:1.991; 95\% Cl: 1.130-3.508; $p=0.017)$ were significantly associated with worse CSS. Additionally, patients with multiple IVR sites also had a shorter survival $(p=0.048)$. Regarding the impact of recurrence time on oncological outcomes, we defined time $\leq 6$ months as the reference juncture, and findings suggest that the HR of CSS significantly decreases between 6 - 24 months (HRD $0.548 ;$; $95 \% \mathrm{Cl}: 0.307-0.980 ; p=0.042$ ) and in the $>24$ months group (HR: $0.129 ; 95 \% \mathrm{Cl}: 0.060-0.278 ; p \rrbracket$ $0.001)$. Using $\leq 59$ years as the reference, we observed a decreased HR for CSS at $\geq 80$ years (HR:2.643, $95 \% \mathrm{Cl}: 1.166-5.989 ; p=0.020)$ patient group. This suggests that the older age range is also an independent predictive factor for poorer CSS. Please see Table 2 for further details.

\section{Discussion}

Despite the fact that UTUC patients with IVR following RNU have poorer CSS, detailed descriptions of the characteristics of secondary $\mathrm{BCa}$ and their influence on post-recurrence outcomes is unknown $[5,6]$. Previous research in this field has to some extent lacked rigour and therefore recommendations remain vague. In order to address this issue and add to the small but growing evidence base around prognostics for patient counselling and follow-up scheduling, we screened and selected a target population by controlling for other known prognostic factors. Using the anonymized, publically available SEER database, we identified and analyzed data from 200 suitable candidates after considering eligibility criteria. While this sample is still relatively small we managed to garner several insights which can be used to guide clinical practice and indeed drive research forward in this field.

Past research has consistently suggested that, tumor stage and lymph node status are the most powerful predictors of survival in UTUC patients [10]. However, with this sample univariable analysis suggests that, both tumor stage for the original UTUC as well as for secondary BCa are important indicators in terms of survival. Although interestingly, the association between UTUC stage and survival is lost and BCa stage becomes the more decisive factor for CSS under multivariable analysis. This assertain can be clearly observed through statistical collinearity between UTUC and BCa staging variables. Although there is one addendum, because this observation may be attributable to the fact that we effectively excluded pT4 and 
lymph node positive patients from this sample. Additionally, the incidence of patients with high stage tumors in this cohort was relatively low. Indeed only one third of this sample (33.5\%) were patients initially assessed as having pT3 tumors. Furthermore, previous literatures have found that the patients with pT0-Ta-T1 carcinomas have similar CSS probabilities compared to patients with pT2 carcinomas $[11,12]$, although this is an area which requires further research.

The other standard pathological feature of secondary $\mathrm{BCa}$ aggressiveness which emerged through this study was tumor grade. According to some literature, low grade BCa poses very low risk to patients in terms of disease progression and CSS [13]. Although, this more general applies to early diagnosis which is not always possible. In high grade tumors, Pietzak et al. found that even Ta and T1 tumors share chromosomal mutations with carcinoma which invade the bladder muscle [14]. This suggests there is likely to be a fundamental difference when it comes to prognosis for BCa patients with different tumor grades. In this study, the advanced grade group also proved to be significantly associated with worse CSS. Therefore, while early diagnosis remains of the utmost importance, we must be particularly vigilant when determining tumor stage and we must discuss with patients further adjuvant therapies such as immediate instillation of intravesical chemotherapy following surgery as a necessary preventative measure.

Several investigators have found a tendency for BCa to be located on or near the resection or BCE site, where the bladder urothelium was injured during cystotomy $[5,15]$. These observations are supported by findings from an in vitro experiment where researchers have found that tumor cells floating in the bladder are more likely to adhere to an injured urothelial surface [2]. However, the research, both basic and clinical, have not always yielded consistent conclusions. For example, Belhadj et al. conducted a large retrospective study which involved 24 centres and 163 patients, in order to assess the distribution of IVR locations following RNU [1]. However, they were unable to identify a trend or even detect a preferred site of recurrence, and our findings appear consistent with this observation. There does not appear to be a significant difference in terms of recurrence rate between surgical and non-surgical locations.

As previously mentioned, detailed information around multiple BCa for this study cohort was not available via the SEER database. Consequently, these patients were categorized as the multiple site group. When exploring the effects of these categories on oncological outcomes, multiple recurrences was determined to be a significant risk factor for worse CSS. Although, this was to be expected because multiplying tumor cells and tumors with multifocal features are of course associated with higher proliferation, migration and the ability to invade vulnerable adjoining locations.

We also found a shorter recurrence time to be associated with worse survival outcome which appears to validate the pathophysiologic mechanism hypothesis previously described. Further support for this can be found in the existing evidence base. For example, Mitra et al. conducted a study which involved 2029 patients in an attempt to identify prognostic factors for survival after urothelial recurrence following radical cystectomy for $\mathrm{BCa}[16]$. The researchers found that patients experiencing early urothelial recurrence often face worse prognosis. This further highlights the aggressive nature of urothelial 
recurrence of urothelial carcinomas and necessitates IVR prevention after RNU. Of course, as always, early detection and even developing effective management strategies for seemingly superficial $\mathrm{BCa}$ might significantly decrease tumor progression [17]. It is beyond the scope of this article to discuss these further although surely this involves raising public awareness and perhaps training general practitioners to inquire when performing standardized health checks which ought to take place regularly.

Interestingly, our findings are line with findings from a 13-center study involving 1453 patients treated with RNU, those aged $\geq 60$ years and also $\geq 80$ years are significantly associated with decreased allcause survival and CSS [18]. Likewise, Chromecki et al. found that patients with an advanced age at the time of RNU are significantly associated with inferior survival after radical surgery [19]. Again predictably, this effect can be attributed to the aging process where co- and indeed multiple morbidities develop, decreasing a patient's natural defense mechanisms. There are a great many unknowns in this situation, all of which influence choice of interventions and care cycles [18,20]. Additionally, biologic changes of the host-tumor relationship in different age groups may also influence survival [21]. Consequently, age ought to be considered when developing adjuvant therapies and patient management schedules.

While this study provided some interesting findings there are several limitations ought to be mentioned. Firstly, this retrospective study summarized data from 9 centres which provide information to the SEER database. This may mean there was selection bias and information biases which may have skewed our findings. Second, as with most previous published studies based upon the SEER database, some limitations could be attributed to the database itself. In this current study for example, necessary information regarding the physical condition, disease focality, the lymph node dissection extent, bladder instillation and other adjuvant treatments after RNU is unavailable through the SEER database. This unfortunately constrained our analysis and therefore has inhibited our ability to provide strong guidelines. It is hoped that as the SEER database develops into a more sophisticated data source, the proprietors will incorporate more necessary variables.

\section{Conclusion}

For primary UTUC patients experiencing IVR after radical surgery, advanced age, multiple sites of IVR, shorter recurrence time, higher $\mathrm{BCa}$ stage and grade were significant independent prognostic factors of poorer CSS. The clinical implications are that we ought to pay more to IVR prevention as well as to earlier signs which may increase the likelihood of early detection. Having the ability to manage what may be seen as the superficial signs of BCa may enable us to improve survival but further research is required.

\section{Abbreviations}

Bladder cuff excision (BCE); Bladder cancer (BCa); Radical nephroureterectomy (RNU); Upper tract urothelial carcinoma (UTUC); Surveillance, Epidemiology, and End Results (SEER) database; Intravesical recurrence (IVR); Interquartile ranges (IQR); Cancer-specific survival (CSS); National Cancer Institute (NCI); Hazard ratio (HR); Confidence interval (Cl); Non-invasive bladder cancer (NIVBC) 


\section{Declarations}

Acknowledgements

None

\section{Author contributions}

Lai Shicong: Conceptualization, Methodology, Formal analysis, Investigation, Writing - original draft.

Wu Pengjie, Liu Jianyong, Liu Shengjie, He Lei: Data curation, Methodology. Software, Investigation.

Seery Samuel: Writing- Reviewing and Editing.

Zhang Yaoguang, Liu Ming, Wang Jianye: Conceptualization, Writing- Reviewing and Editing, Project administration, Supervision. All authors read and approved the final manuscript.

\section{Funding}

This work was supported by the Beijing Municipal Science and Technology Project (Grant number: Z201100005620007); the Beijing Hospital Clinical Research 121 Project (Grant number: BJ-2018-090); the Beijing Hospital Clinical Research (Grant number: BJ-2015-098) and the National Key R\&D Program of China [Grant Numbers: 2018YFC2002202].

\section{Availability of data and materials}

The data of the current study are extracted from the SEER database.

\section{Ethical approval}

Since the SEER database is publicly accessible and patient information was anonymous, this study does not require informed consent. However, current study conformed to the 1964 Helsinki Declaration (and the more recent amendments) and was performed in accordance with the ethical standards of the institutional and national research committee.

\section{Consent for publication}

The data of the current study are extracted from the SEER database. No need written informed consent.

\section{Competing interests}

The authors' declare that they have no competing interests.

\section{References}


1 Belhadj Y, Grande P, Seisen T et al. Bladder mapping of tumour recurrence after radical nephroureterectomy for upper tract urothelial carcinoma and its influence on oncological outcomes. BJU Int 2019; 123: 618-23.

2 Hafner C, Knuechel R, Stoehr R, Hartmann A. Clonality of multifocal urothelial carcinomas: 10 years of molecular genetic studies. Int J Cancer 2002; 101: 1-6.

3 Milojevic B, Dzamic Z, Kajmakovic B, Milenkovic Petronic D, Sipetic Grujicic S. Urothelial carcinoma: Recurrence and risk factors. J BUON2015; 20: 391-8.

4 Xylinas E, Colin P, Audenet F et al. Intravesical recurrence after radical nephroureterectomy for upper tract urothelial carcinomas: predictors and impact on subsequent oncological outcomes from a national multicenter study. World J Urol 2013; 31: 61-8.

5 Yamashita S, Ito A, Mitsuzuka K et al. Clinical implications of intravesical recurrence after radical nephroureterectomy for upper urinary tract urothelial carcinoma. Int J Urol 2016; 23: 378-84.

6 Elalouf V, Xylinas E, Klap J et al. Bladder recurrence after radical nephroureterectomy: predictors and impact on oncological outcomes. Int J Urol 2013; 20: 1078-83.

7 Hou G, Zheng Y, Zhang L et al. Development and validation of a prognostic nomogram for patients with intravesical recurrence after radical nephroureterectomy for non-metastatic upper tract urothelial carcinoma. World J Urol 2020; 38: 1969-75.

8 Kang M, Jeong CW, Kwak C, Kim HH, Ku JH. The characteristics of recurrent upper tract urothelial carcinoma after radical nephroureterectomy without bladder cuff excision. Yonsei Med J 2015; 56: 37581.

9 Nazzani S, Preisser F, Mazzone E et al. Nephroureterectomy with or without Bladder Cuff Excision for Localized Urothelial Carcinoma of the Renal Pelvis. Eur Urol Focus 2020; 6: 298-304.

10 Chromecki TF, Bensalah K, Remzi M et al. Prognostic factors for upper urinary tract urothelial carcinoma. Nat Rev Urol 2011; 8: 440-7.

11 Novara G, De Marco V, Gottardo F et al. Independent predictors of cancer-specific survival in transitional cell carcinoma of the upper urinary tract: multi-institutional dataset from 3 European centers. Cancer 2007; 110: 1715-22.

12 Abouassaly R, Alibhai SM, Shah N, Timilshina N, Fleshner N, Finelli A. Troubling outcomes from population-level analysis of surgery for upper tract urothelial carcinoma. Urology 2010; 76: 895-901.

13 Matulay JT, Soloway M, Witjes JA et al. Risk-adapted management of low-grade bladder tumours: recommendations from the International Bladder Cancer Group (IBCG). BJU Int 2020; 125: 497-505. 
14 Pietzak EJ, Bagrodia A, Cha EK et al. Next-generation Sequencing of Nonmuscle Invasive Bladder Cancer Reveals Potential Biomarkers and Rational Therapeutic Targets. Eur Uro/ 2017; 72: 952-9.

$15 \mathrm{Kang} \mathrm{CH}, \mathrm{Yu} \mathrm{TJ}, \mathrm{Hsieh} \mathrm{HH}$ et al. The development of bladder tumors and contralateral upper urinary tract tumors after primary transitional cell carcinoma of the upper urinary tract. Cancer 2003; 98: 1620-6.

16 Mitra AP, Alemozaffar M, Harris BN, Schuckman AK, Skinner EC, Daneshmand S. Outcomes after urothelial recurrence in bladder cancer patients undergoing radical cystectomy. Urology 2014; 84: 1420-6.

17 Shigeta K, Kikuchi E, Hagiwara M et al. The Conditional Survival with Time of Intravesical Recurrence of Upper Tract Urothelial Carcinoma. J Urol 2017; 198: 1278-85.

18 Shariat SF, Godoy G, Lotan Y et al. Advanced patient age is associated with inferior cancer-specific survival after radical nephroureterectomy. BJU Int 2010; 105: 1672-7.

19 Chromecki TF, Ehdaie B, Novara G et al. Chronological age is not an independent predictor of clinical outcomes after radical nephroureterectomy. World J Urol 2011; 29: 473-80.

20 Yancik R, Havlik RJ, Wesley MN et al. Cancer and comorbidity in older patients: a descriptive profile. Ann Epidemiol 1996; 6: 399-412.

21 Taccoen X, Valeri A, Descotes $\mathrm{JL}$ et al. Renal cell carcinoma in adults 40 years old or less: young age is an independent prognostic factor for cancer-specific survival. Eur Uro/ 2007; 51: 980-7.

\section{Tables}

Table 1. Characteristics of patients with intravesical recurrence following radical nephroureterectomy for non-metastatic upper tract urothelial carcinoma 


\begin{tabular}{|c|c|}
\hline \multicolumn{2}{|l|}{ Variables } \\
\hline \multicolumn{2}{|l|}{ Age in years, $n(\%)$} \\
\hline$\otimes 59$ & $36(18)$ \\
\hline $60-79$ & $115(57.5)$ \\
\hline$\geq 80$ & $49(24.5)$ \\
\hline \multicolumn{2}{|l|}{ Gender, n (\%) } \\
\hline Male & $113(56.5)$ \\
\hline Female & $87(43.5)$ \\
\hline \multicolumn{2}{|l|}{ Race, n (\%) } \\
\hline White & $182(91)$ \\
\hline Black & $6(3)$ \\
\hline Other & $12(6)$ \\
\hline \multicolumn{2}{|l|}{ UTUC Laterality, n (\%) } \\
\hline Left & $103(51.5)$ \\
\hline Right & $97(47.5)$ \\
\hline \multicolumn{2}{|l|}{ UTUC Location, n (\%) } \\
\hline Pelvis & $132(66)$ \\
\hline Ureter & $68(34)$ \\
\hline \multicolumn{2}{|l|}{ UTUC stage, n (\%) } \\
\hline pTa-1 & $90(45)$ \\
\hline pT2-3 & $110(55)$ \\
\hline \multicolumn{2}{|l|}{ Histopathological UTUC, n (\%) } \\
\hline Low (Well and moderately differentiated) & $49(24.5)$ \\
\hline High (Poorly diferentiated and undiferentiated) & $151(75.5)$ \\
\hline \multicolumn{2}{|l|}{ Secondary BCa location, n (\%) } \\
\hline Non-surgical Site (bladder dome, neck or later wall) & $42(21)$ \\
\hline Surgical site (bladder trigone, anterior or posterior wall) & $53(26.5)$ \\
\hline Multiple site (multifocal tumors) & $105(52.5)$ \\
\hline Secondary BCa stage, n (\%) & \\
\hline
\end{tabular}




\begin{tabular}{|ll|}
\hline pTa-1 & $180(90)$ \\
\hline pT2-4 & $20(10)$ \\
\hline Histopathological BC grade, $\mathbf{n}(\%)$ & $82(41)$ \\
\hline Low (Well and moderately differentiated) & $118(59)$ \\
\hline High (Poorly diferentiated and undiferentiated) & \\
\hline Recurrence time in months, $\mathbf{n}(\%)$ & $53(26.5)$ \\
\hline Time $\leq 6$ & $74(37)$ \\
\hline $6 \square T i m e \leq 24$ & $73(36.5)$ \\
\hline Time>24 & $71(36,103.75)$ \\
\hline Follow-up in months, median (IQR) & \\
\hline BCa = bladder cancer; p T = pathological tumour; UTUC = upper tract urothelial carcinoma
\end{tabular}

Table 2. Univariate and multivariate Cox regression analyses predicting CSS for patients with IVR following RNU for non-metastatic UTUC 


\begin{tabular}{|c|c|c|c|c|c|c|}
\hline \multirow[t]{2}{*}{ Variables } & \multicolumn{3}{|c|}{ Univariate } & \multicolumn{3}{|c|}{ Multivariate } \\
\hline & $\mathrm{HR}$ & $95 \% \mathrm{Cl}$ & $\begin{array}{l}\mathrm{p} \\
\text { value }\end{array}$ & $\mathrm{HR}$ & $95 \% \mathrm{Cl}$ & $\begin{array}{l}p \\
\text { value }\end{array}$ \\
\hline Age in years & & & 0.006 & & & 0.021 \\
\hline$\otimes 59$ & 1 & Reference & & 1 & Reference & \\
\hline $60-79$ & 1.188 & $\begin{array}{l}0.565- \\
2.497\end{array}$ & 0.650 & 1.298 & $\begin{array}{l}0.607- \\
2.774\end{array}$ & 0.501 \\
\hline$\geq 80$ & 2.721 & $\begin{array}{l}1.236- \\
5.990\end{array}$ & 0.013 & 2.643 & $\begin{array}{l}1.166- \\
5.989\end{array}$ & 0.020 \\
\hline Gender & & & & - & - & - \\
\hline Male & 1 & Reference & & & & \\
\hline Female & 1.213 & $\begin{array}{l}0.733- \\
2.008\end{array}$ & 0.453 & & & \\
\hline Race & & & 0.401 & & & \\
\hline White & 1 & Reference & & & & \\
\hline Black & 0.479 & $\begin{array}{l}0.066- \\
3.461\end{array}$ & 0.465 & & & \\
\hline Other & 0.436 & $\begin{array}{l}0.106- \\
1.784\end{array}$ & 0.248 & - & - & \\
\hline \multicolumn{7}{|l|}{ UTUC laterality } \\
\hline Left & 1 & Reference & & & & \\
\hline Right & 1.029 & $\begin{array}{l}0.622- \\
1.701\end{array}$ & 0.911 & & & \\
\hline \multicolumn{7}{|l|}{ UTUC location } \\
\hline Pelvis & 1 & Reference & & & & \\
\hline Ureter & 1.362 & $\begin{array}{l}0.820- \\
2.263\end{array}$ & 0.233 & & & \\
\hline \multicolumn{7}{|l|}{ UTUC stage } \\
\hline pTa-1 & 1 & Reference & & & & \\
\hline pT2-3 & 1.872 & $\begin{array}{l}1.095- \\
3.200\end{array}$ & 0.022 & & & \\
\hline \multicolumn{7}{|c|}{ Histopathological UTUC grade } \\
\hline $\begin{array}{l}\text { Low (Well and moderately } \\
\text { differentiated) }\end{array}$ & 1 & Reference & & & & \\
\hline
\end{tabular}




\begin{tabular}{|c|c|c|c|c|c|c|}
\hline $\begin{array}{l}\text { High (Poorly differentiated and } \\
\text { undifferentiated) }\end{array}$ & 1.764 & $\begin{array}{l}0.918- \\
3.391\end{array}$ & 0.089 & & & \\
\hline Secondary BCa location & & & 0.033 & & & 0.048 \\
\hline Non-surgical Site & 1 & Reference & & 1 & Reference & \\
\hline Surgical site & 0.376 & $\begin{array}{l}0.168- \\
0.840\end{array}$ & 0.017 & 0.539 & $\begin{array}{l}0.238- \\
1.221\end{array}$ & 0.539 \\
\hline Multiple site (multifocal tumors) & 0.590 & $\begin{array}{l}0.343- \\
1.018\end{array}$ & 0.058 & 0.505 & $\begin{array}{l}0.287- \\
0.889\end{array}$ & 0.018 \\
\hline \multicolumn{7}{|l|}{ Secondary BCa stage } \\
\hline pTa-1 & 1 & Reference & & 1 & Reference & \\
\hline pT2-4 & 2.441 & $\begin{array}{l}1.267- \\
4.702\end{array}$ & 0.008 & 2.900 & $\begin{array}{l}1.441- \\
5.839\end{array}$ & 0.003 \\
\hline \multicolumn{7}{|l|}{ Histopathological grade of $\mathrm{BCa}$} \\
\hline $\begin{array}{l}\text { Low (Well and moderately } \\
\text { differentiated) }\end{array}$ & 1 & Reference & & 1 & Reference & \\
\hline $\begin{array}{l}\text { High (Poorly diferentiated and } \\
\text { undiferentiated) }\end{array}$ & 1.984 & $\begin{array}{l}1.141- \\
3.449\end{array}$ & 0.015 & 1.991 & $\begin{array}{l}1.130- \\
3.508\end{array}$ & 0.017 \\
\hline Recurrence time, months & & & 0.001 & & & 0.001 \\
\hline Time $\leq 6$ & 1 & Reference & & 1 & Reference & \\
\hline $6 \bigotimes \mathrm{Time} \leq 24$ & 0.567 & $\begin{array}{l}0.326- \\
0.989\end{array}$ & 0.045 & 0.548 & $\begin{array}{l}0.307- \\
0.980\end{array}$ & 0.042 \\
\hline Time>24 & 0.184 & $\begin{array}{l}0.090- \\
0.375\end{array}$ & 0.001 & 0.129 & $\begin{array}{l}0.060- \\
0.278\end{array}$ & 0.001 \\
\hline \multicolumn{7}{|c|}{$\begin{array}{l}\mathrm{CSS}=\text { cancer-specific survival } \llbracket \mathrm{p} \mathrm{T}=\text { pathological tumour; } \mathrm{HR}=\text { hazard ratio; } \mathrm{Cl}=\text { confidence interval; } \\
\mathrm{BCa}=\text { bladder cancer; }\end{array}$} \\
\hline $\begin{array}{l}\mathrm{RNU}=\text { radical nephroureterectom } \\
\text { recurrence }\end{array}$ & uppe & inary trac & heli & arcir & IVR = in & sical \\
\hline
\end{tabular}

\section{Figures}


A

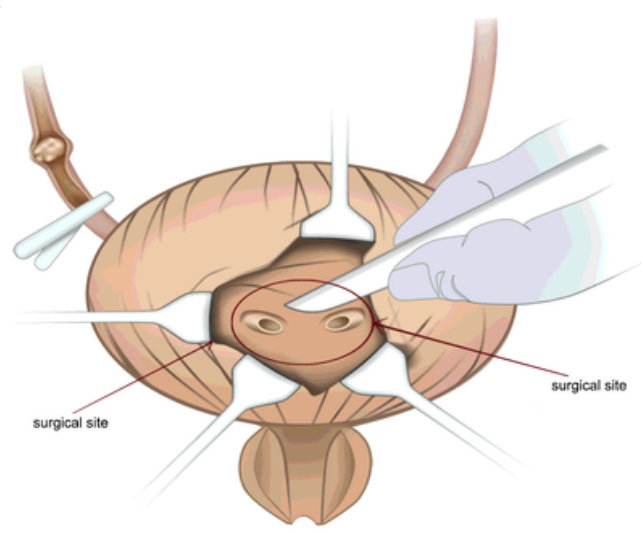

B

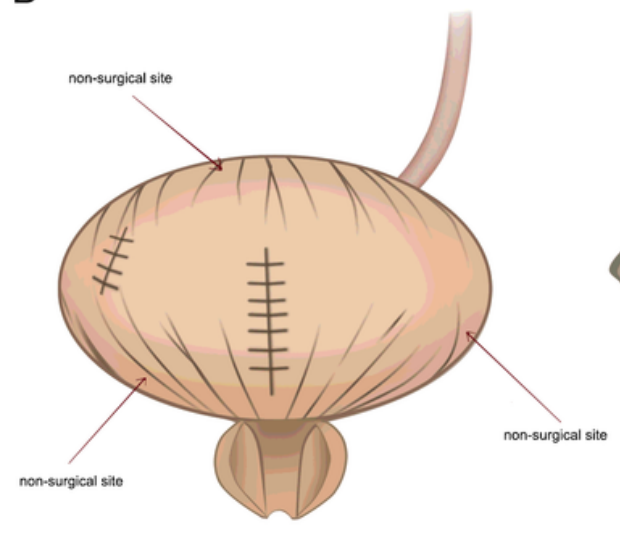

C

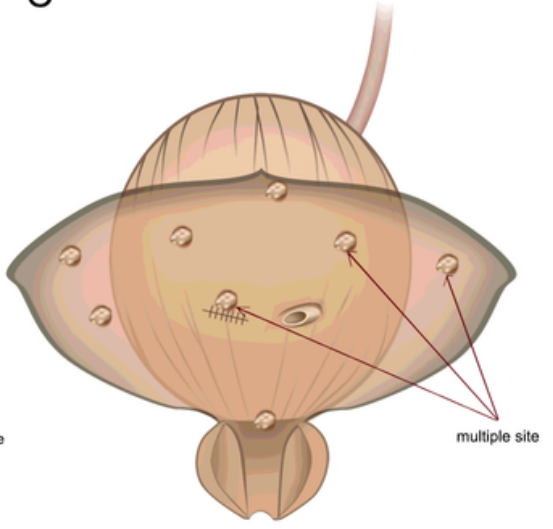

Figure 1

(A) An overview of the surgical site of the bladder (i.e, the bladder trigone or posterior wall) and/or open excision filed (anterior wall); (B) An overview of non-surgical site of the bladder (i.e, bladder dome, neck or later wall); (C) An overview of the multiple bladder recurrence site. 
A
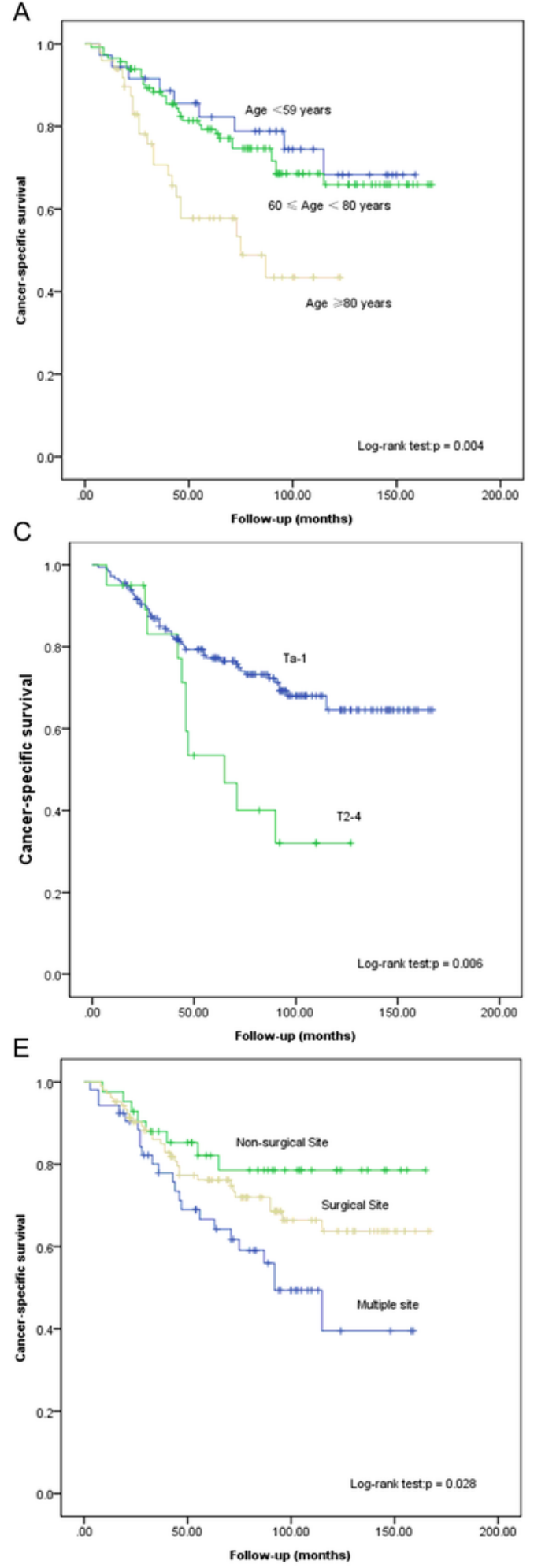

B

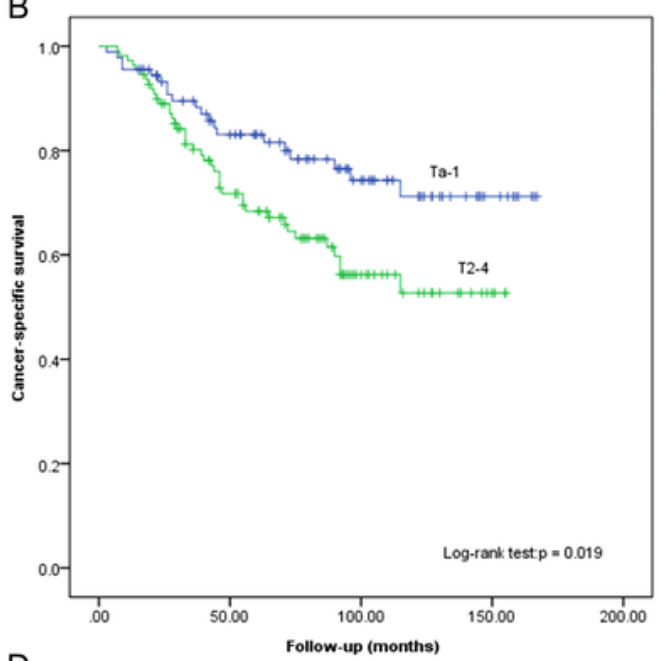

$\mathrm{D}$

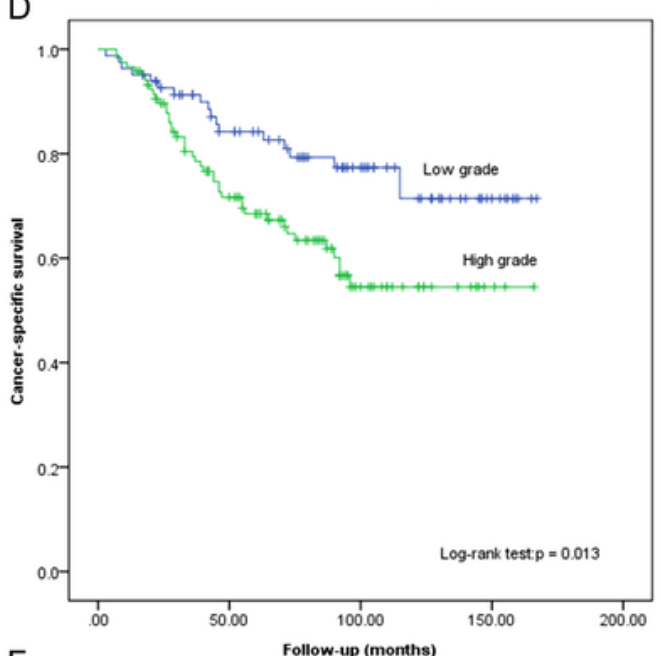

$\mathrm{F}$

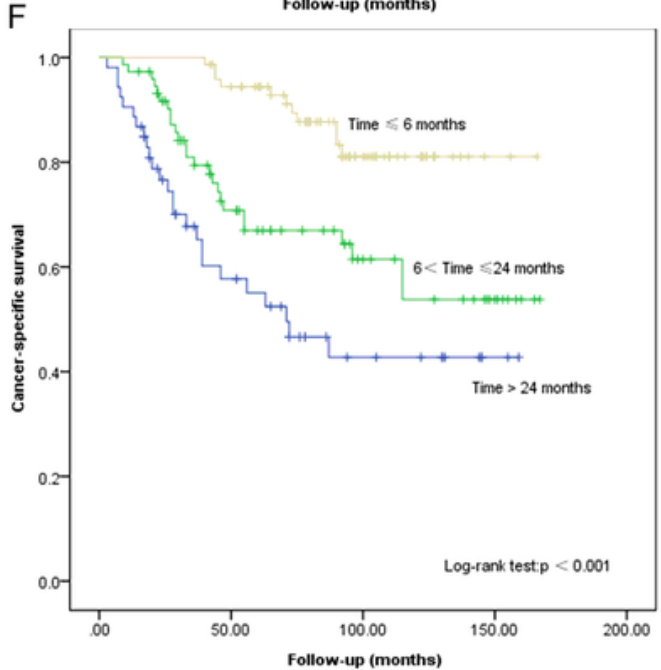

Figure 2

Cancer-specific survival of patients with intravesical recurrence following radical nephroureterectomy stratified by (A) Age, (B) UTUC stage, (C) BCa stage, (D) BCa grade, (E) BCa location, and (F) Recurrence time. (UTUC=upper urinary tract urothelial carcinoma; $\mathrm{BCa}=$ bladder cancer) 